\title{
Pharmacokinetics and Biodistribution of Human Serum Albumin-TIMP-2 Fusion Protein Using Near-Infrared Optical Imaging
}

\author{
Mi-Sook Lee ${ }^{a, d}$, Young Han Kim ${ }^{a}$, Yeon Joo Kim ${ }^{a}$, Seung-Hae Kwon ${ }^{a}$, Jeong-kyu Bang ${ }^{a}$, Sang-Mok Lee ${ }^{b}$, Yun Seon Song ${ }^{c}$, \\ Dae-Hyun Hahm ${ }^{\mathrm{d}}$, Insop Shim ${ }^{\mathrm{d}}$, Daeseok Han ${ }^{\mathrm{e}}$ and Song Her ${ }^{\mathrm{a}}$ \\ ${ }^{a}$ Division of Bio-Imaging, Chuncheon Center, Korea Basic Science Institute, Chuncheon 200-701,Republic of Korea. \\ ${ }^{\mathrm{b}}$ BiocurePham, Daejeon Bio Venture Town, Daejeon 305-811, Republic of Korea.

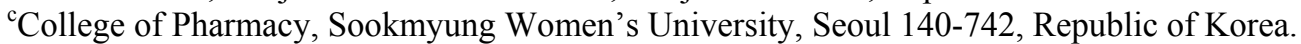 \\ ${ }^{\mathrm{d}}$ Acupuncture and Meridian Science Research Center, College of Oriental Medicine, Kyung Hee University, Seoul, 130- \\ 701, Korea. \\ ${ }^{\mathrm{e}}$ Korea Food Research Institute, Seongnam 463-746, Republic of Korea
}

Received, June 9, 2011; Revised, August 12, 2011; Accepted, September 20, 2011; Published, September $26,2011$.

\begin{abstract}
Purpose TIMP-2 has been studied as an attractive cancer therapeutic candidate, and a TIMP-2 fusion protein (HSA/TIMP-2) displayed effective anticancer activity, despite a lack of information about its pharmacokinetics (PK) and biodistribution. The purpose of this work was to assess the PK and biodistribution of HSA/TIMP-2 as well as to quantify accumulated HSA/TIMP-2 in tumors. Methods Cy5.5 near-infrared (NIR) fluorescence was conjugated to the HSA/TIMP-2 protein (Cy5.5-HSA/TIMP-2) for monitoring spatio-temporal changes in vivo. For PK and biodistribution analysis, $0.2 \mu \mathrm{g} / \mathrm{g}$ body weight of Cy5.5-HSA/TIMP-2 was injected into MAT-LyLu prostate tumor xenografts, which were then imaged using an IVIS-200 optical imaging system. To quantify the accumulated HSA/TIMP-2 in tumors, we introduced a standard curve with depth-corrected fluorescence measurement. Results In the vascular tube formation assay with human umbilical vein endothelial cells (HUVECs), Cy5.5-HSA/TIMP-2 showed an antiangiogenic effect. In prostate cancer xenografts, Cy5.5HSA/TIMP-2 exhibited a prolongation of blood half-life to $19.6 \mathrm{~h}$ and relatively preferential distribution to the tumor. The amount of tumor-accumulated Cy5.5-HSA/TIMP-2 was calculated to be $4.5 \pm 0.5 \mathrm{ng} / \mathrm{g}$ body weight at 2 days, representing $2.25 \pm 0.25 \%$ of the initial dose. Conclusions We evaluated the pharmacokinetic profile and biodistribution of HSA/TIMP-2 with favorable results, providing new information for more effective approaches to cancer therapeutics using HSA/TIMP-2. Additionally, real-time in vivo fluorescence imaging analysis using a depth-corrected standard curve may serve as a platform to quantify biodistributed drug in anticancer therapeutic studies.
\end{abstract}

This article is open to POST-PUBLICATION REVIEW. Registered readers (see "For Readers") may comment by clicking on ABSTRACT on the issue's contents page.

\section{INTRODUCTION}

TIMP-2 is a natural inhibitor of matrix metalloproteinase-2 and has been studied as an attractive cancer therapeutic candidate (1-3). Many $T I M P-2$ gene therapy studies using adenovirus and retrovirus delivery systems have supported the role of TIMP-2 in suppressing angiogenesis and consequent tumor growth in murine cancer models $(4,5)$. However, virus-mediated gene therapy in humans is still limited by the safety of the therapeutic application. The use of high-titer adenovirus to achieve acceptable gene expression has also been frequently associated with cytotoxicity and immune responses, and retrovirus was reported to be the causative agent of breast cancer. One way to overcome the limitation of virus-mediated gene therapy is the use of recombinant technology to express target therapeutic proteins. Although human TIMP-2 is a non-glycosylated protein and could be expressed in Escherichia coli, protein insolubility still remains the main problem because of the aggregation of incorrectly folded molecules, which in turn may be (6). Furthermore, the time-consuming and laborious refolding process could not produce proper amounts of active TIMP-2 protein. The human TIMP-2 gene

Corresponding Author: Song Her, Ph.D.; Division of BioImaging, Chuncheon Center; Korea Basic Science Institute; Chuncheon 200-701, Korea; E-mail: swher@kbsi.re.kr 
due to the presence of incorrect disulfide bridges was cloned into a yeast expression vector to produce a high-expression eukaryotic system; however, expression of TIMP-2 proteins was barely detected in transformed yeast Saccharomyces cerevisiae. Taking advantage of human serum albumin (HSA) fusion technology, active HSA/TIMP-2 fusion protein was expressed with high-yield production in the culture broth and showed effective anticancer activity (7). However, little is known about the pharmacokinetics (PK) and biodistribution aspects of this protein, which are important parameters for the design and testing of a drug in vivo.

Traditionally, PK and biodistribution studies were based either on quantification of the therapeutic agent using high-performance liquid chromatography (HPLC) and mass spectroscopy or on measuring radioactivity in homogenized tissue after radiolabeling the agent $(8,9)$. Both approaches are time consuming, relatively expensive, and outside the area of the expertise of many protein purification laboratories (10). Alternatively, optical imaging using a near-infrared (NIR) fluorescent probe in living animals has become an important tool for evaluation of PK and biodistribution, with advantages of fast and convenient detection (11, 12). However, non-invasive fluorescence imaging fails to reflect quantitative data accurately because detection of fluorescence intensity is strongly dependent on the tissue lesion depth and optical properties of the lesion $(12,13)$. Thus, spatial quantification is needed to compensate for the loss of imaging signal. In the present study, we evaluated PK and biodistribution parameters using a Cy5.5-conjugated HSA/TIMP-2, and we also quantified the HSA/TIMP-2 accumulated in tumors based on a standard curve.

Here, using NIR-based technology, we evaluate the PK and biodistribution of HSA/TIMP-2 and also describe a new, fast, and economical method for semi-quantification of accumulated HSA/TIMP2 in tumors.

\section{MATERIALS AND METHODS}

\section{Cell culture}

MAT-LyLu rat prostate adenocarcinoma cells (MLL; American Type Culture Collection, Rockville, MD, USA) were cultured in Dulbecco's modified Eagle's medium (DMEM; Lonza, Walkersville, MD, USA) supplemented with $10 \%$ (v/v) fetal bovine serum (Hyclone, Logan, UT, USA).

Human umbilical vein endothelial cells (HUVECs) were kindly supplied by Dr. KwonSoo Ha's group at Kangwon University, Korea. HUVECs were cultured in growth medium 199 (Gibco-BRL, Grand Island, NY, USA) supplemented with $20 \%$ fetal bovine serum and 10 $\mathrm{ng} / \mathrm{mL}$ human basic fibroblast growth factor (bFGF; R\&D Systems, Inc., Minneapolis, MN, USA). Confluent HUVECs (passages 4-7) were used for the experiments.

\section{In vitro tube formation assay}

Tube formation of HUVECs on Matrigel was performed by procedures described previously (7). Briefly, Matrigel (Becton Dickinson, Bedford, MA, USA) was added to a 24-well plate and allowed to polymerize at $37^{\circ} \mathrm{C}$ for $1 \mathrm{~h}$. HUVECs $\left(1 \times 10^{5}\right.$ cells/well) were seeded in the Matrigel-coated well and incubated for $6 \mathrm{~h}$. In vitro tube formation was imaged under a phase-contrast microscope.

\section{Conjugation of Cy5.5 to HSA/TIMP-2}

HSA/TIMP-2 was conjugated to Cy5.5 according to the manufacturer's recommended protocol. Briefly, a reaction between HSA/TIMP-2 and the Cy5.5NHS ester (Invitrogen, Carlsbad, CA, USA) at a molar ratio of 1:2 was performed in the dark at room temperature for $1 \mathrm{~h}$. Unconjugated dye was removed by dialysis against phosphate-buffered saline (PBS) using a Slide-A-Lyzer membrane cassette (3,500 MWCO; Pierce Biotechnology, Rockford, IL, USA) for up to $18 \mathrm{~h}$ at $4^{\circ} \mathrm{C}$. Dialyzed samples were filtered through a $0.2-\mu \mathrm{m}$ syringe filter, separated by sodium dodecyl sulfatepolyacrylamide gel electrophoresis (SDS-PAGE), and analyzed using the Xenogen IVIS-200 imaging system (Xenogen, Alameda, CA, USA) to ensure quality before use.

\section{Measurement of blood clearance}

Male BALB/c nude mice (8-10 weeks old; Orient, Gyeonggi-do, Korea) were administered a single tail-vein injection of $0.2 \mu \mathrm{g} / \mathrm{g}$ body weight of Cy5.5-HSA/TIMP-2 or an equivalent amount of Cy5.5, and serum samples were then collected at various time points thereafter by tail nicking. Cy5.5 fluorescence intensity was measured using a Xenogen IVIS-200 imaging system (Xenogen), and its half-life in the circulation was calculated (GraphPad Prism; GraphPad Software, Inc., San 
Diego, CA, USA). The use and care of animals were reviewed and approved by the Institutional Animal Care and Use Committee of the Korea Basic Science Institute.

\section{Subcutaneous tumor xenograft and whole-body NIR imaging}

To establish the subcutaneous xenograft model, $1 \times$ $10^{5}$ MLL cells were subcutaneously (sc) injected into the right dorsal flank of $\mathrm{BALB} / \mathrm{c}$ nude mice (14). When the tumor volume reached approximately $250 \mathrm{~mm}^{3}, 0.2 \mu \mathrm{g} / \mathrm{g}$ body weight of Cy5.5-HSA/TIMP-2 was administered by intraperitoneal (ip) injection, and Cy5.5 fluorescence was then monitored for up to 7 days after administration. For biodistribution analysis, organs were imaged immediately following dissection 2 days post-administration of Cy5.5HSA/TIMP-2, and fluorescence intensity (photons/second $(\mathrm{p} / \mathrm{s})$ ) was calculated per wet weight of each organ (15). All images were acquired for $\sim 5 \mathrm{~s}$ and were processed using the Xenogen Living Image software (16).

\section{Fluorescence confocal microscopy}

A confocal laser scanning microscope (LSM510 Meta; Carl Zeiss, Oberkochen, Germany) equipped with $\times 20$ dry and $\times 100$ oil objectives was used to collect tissue images. To visualize Cy5.5HSA/TIMP-2, tumor tissues were removed and then cryosectioned at $8-\mu \mathrm{m}$ slice thickness. Slices from tumor tissue were stained with Hoechst 33342 (Molecular Probes, Inc., Eugene, USA) for $10 \mathrm{~min}$ at room temperature. The tissue was then excited using multiple color laser lines (405 $\mathrm{nm}$ for Hoechst and $643 \mathrm{~nm}$ for Cy5.5), and the emission was collected through appropriate narrow band-pass filters.

\section{Statistical analyses}

For all results, values are expressed as the means \pm standard error of the mean (SEM). Statistical analyses were performed using Student's $t$-test, unless indicated otherwise. Differences were considered statistically significant at $* P<0.05$ and $* * P<0.01$.

\section{RESULTS}

Characterization and blood half-life of Cy5.5HSA/TIMP-2

To determine which HSA/TIMP-2 proteins were conjugated with $\mathrm{Cy} 5.5$, labeled and nonlabeled HSA/TIMP-2 was resolved in SDS-PAGE gel. Protein separation showed a single band at around $88 \mathrm{kDa}$ of Cy5.5-conjugated HSA/TIMP-2 (Fig. $1 \mathrm{~B}-\mathrm{a})$, and a fluorescent signal was detected only from Cy5.5-HSA/TIMP-2 without free Cy5.5 (Fig. 1B-b). The fluorescence intensity also showed a strong correlation with Cy5.5-HSA/TIMP-2 concentration $\left(R^{2}=0.98\right.$; Fig. $\left.1 \mathrm{C}\right)$. To investigate whether Cy5.5 conjugation altered the antiangiogenic activity of HSA/TIMP-2, a vascular tube formation assay with HUVECs was performed. In the morphogenic assay on Matrigel, the capillary-like tubular structure of the HUVEC was completely disrupted at $10 \mu \mathrm{M}$ Cy5.5-HSA/TIMP2 (Figure 1D). These results suggest that Cy5.5 conjugation to HSA/TIMP-2 did not alter the antiangiogenic activity, confirming that NIR Cy5.5labeling was suitable for the present study. Next, we examined the blood half-life by measuring fluorescence intensities of blood samples obtained by tail nicking at the times indicated. As shown in Figure 1E, the Cy5.5-HSA/TIMP-2 exhibited a prolonged circulatory half-life of $19.6 \pm 3.2 \mathrm{~h}$, compared with $2.2 \pm 0.7 \mathrm{~h}$ for Cy5.5.

\section{High accumulation of Cy5.5-HSA/TIMP-2 in tumors}

To analyze the distribution of Cy5.5-HSA/TIMP-2, a single ip injection of Cy5.5-HSA/TIMP-2 was administered to mice bearing MLL tumors, and whole-body imaging was conducted. One day after the injection, fluorescence from Cy5.5-HSA/TIMP2 was detected throughout the animals' bodies, whereas fluorescent signals were rarely detected in free Cy5.5-treated tumor xenografts (Fig. 2A). At day 2, the fluorescent signal from Cy5.5HSA/TIMP-2 showed a peak within the tumor (Fig. 2B), revealing a time-dependent accumulation in the tumor. Consistent with the whole-body biophotonic images, relatively high accumulation in tumor tissue was observed in the ex vivo distribution images of organs (Fig. 2C, D). 
A

124609

\begin{tabular}{l|l}
\hline $\begin{array}{l}\text { Signal } \\
\text { peptide }\end{array}$ & HSA $(67 \mathrm{kDa}) \quad$ TIMP-2 (21 kDa)
\end{tabular}

B

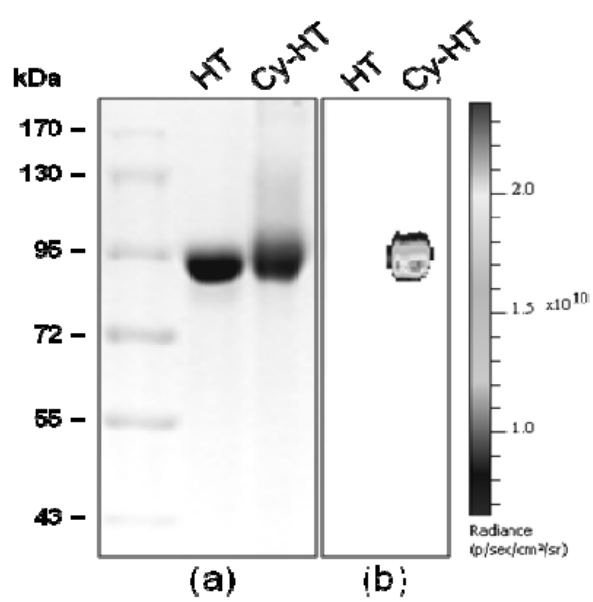

$\mathbf{C}$

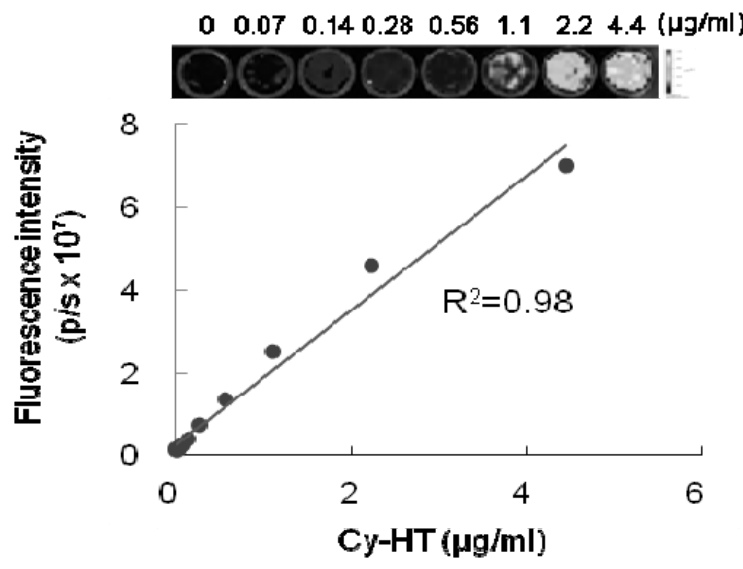

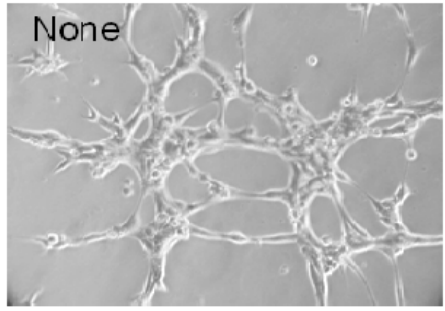
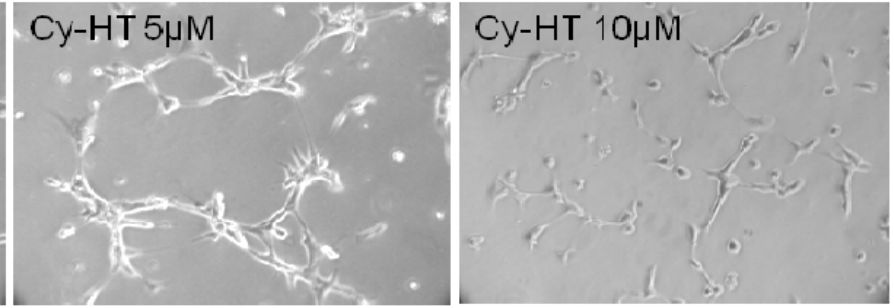

E

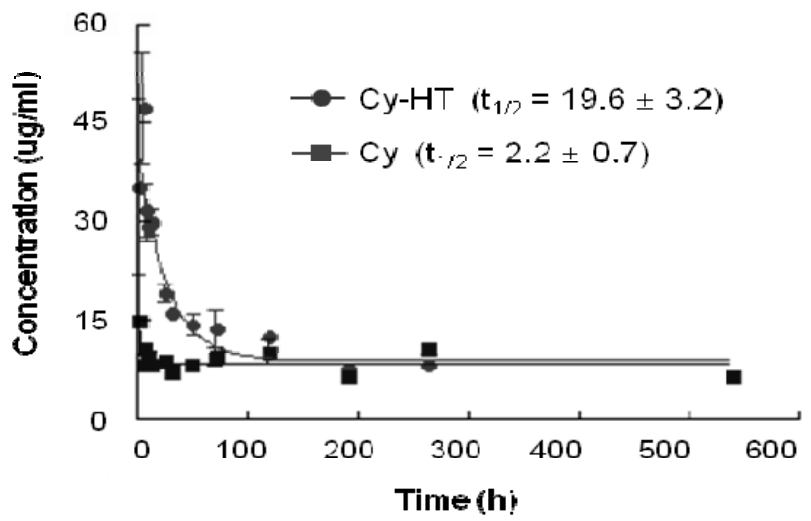

Figure 1. Characterization and blood half-life of Cy5.5-HSA/TIMP-2. (A) Schematic diagram of HSA/TIMP-2. (B) HSA/TIMP-2 and Cy5.5-HSA/TIMP-2 were separated by SDS-PAGE and then visualized using Coomassie Brilliant Blue $\mathrm{R}$ (a) or the IVIS-200 imaging system (b). (C) Correlation between fluorescence intensity ( $Y$-axis) and the concentration $(X$ axis) of Cy5.5-HSA/TIMP-2. Spearman's rank correlation coefficient $\left(R^{2}=0.98\right)$ is indicated. (D) Microscopic images of HUVEC tube formation on Matrigel. One hundred thousand HUVECs were seeded onto Matrigel in the presence of Cy5.5HSA/TIMP-2 and incubated for $6 \mathrm{~h}$. Capillary-like tube formation in the HUVECs was also completely inhibited by $10 \mu \mathrm{M}$ Cy5.5-HSA/TIMP-2. The pictures were taken at an original magnification of 200×. (E) Measured fluorescence intensity of Cy5.5-HSA/TIMP-2 in serum. The blood circulation half-life was determined using the bi-exponential nonlinear regression function in GraphPad Prism software. Data points represent the means \pm SEM ( $n=4$ mice per time point). HT, HSA/TIMP2; Cy-HT, Cy5.5-HSA/TIMP-2. 
A

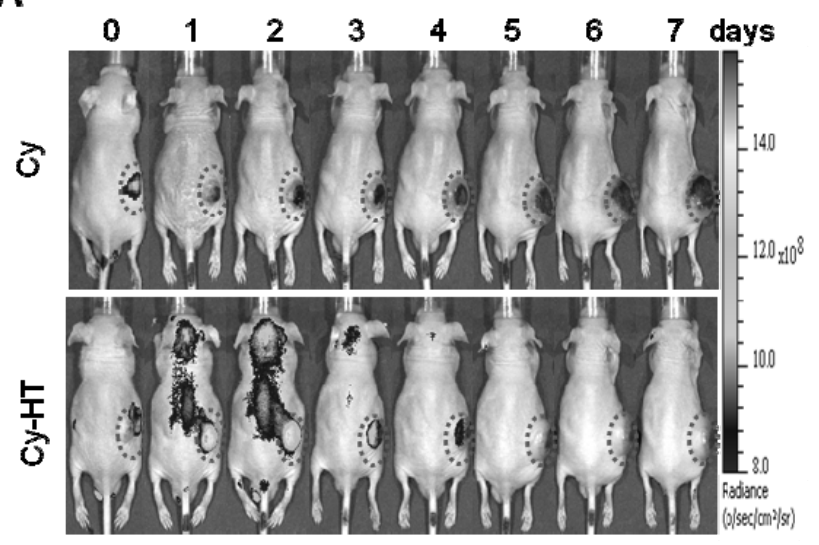

B

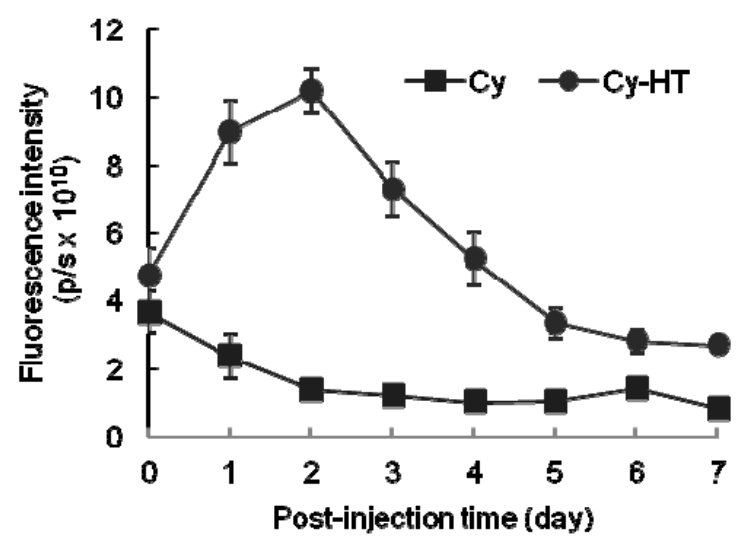

C

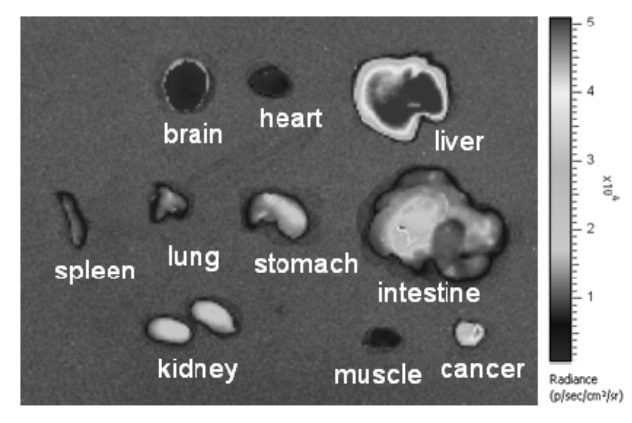

D

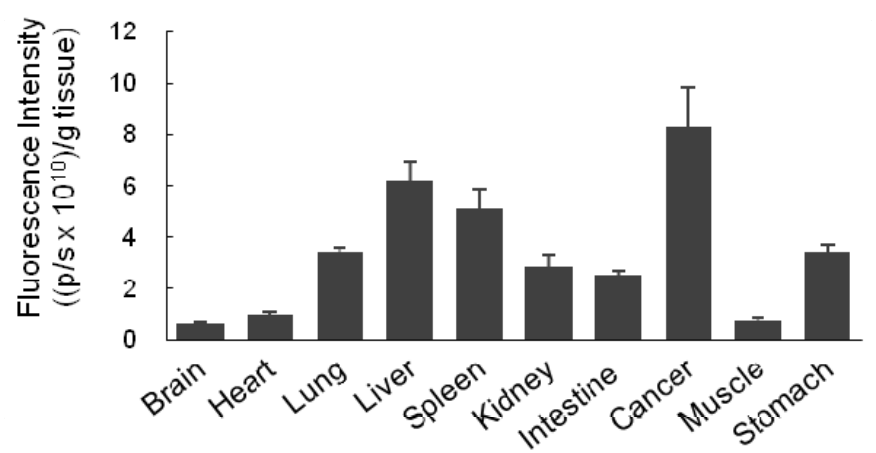

Figure 2. Biodistribution of Cy5.5-HSA/TIMP-2 in the whole body and organs. (A) Representative images of the whole body and (B) quantitation of fluorescent signal in the tumor. Noninvasive fluorescence images were obtained in MLL tumor-bearing mice after intraperitoneal (ip) injection with $100 \mu \mathrm{L}$ of $0.2 \mu \mathrm{g} / \mathrm{g}$ body weight of HSA/TIMP-2-Cy5.5. The fluorescence intensity of the tumor was quantified based on the total photon count determined from regions of interest analysis. Each data point represents the mean \pm SEM $(n=4)$. (C) Representative fluorescence image and (D) fluorescence intensities of dissected organs. The organs were imaged immediately following dissection 2 days after ip administration and calculated as fluorescence intensity (photons/second $(\mathrm{p} / \mathrm{s})$ ) per wet weight of each organ. Each data point represents the mean \pm SEM $(n=4)$. HT, HSA/TIMP-2; Cy-HT, Cy5.5-HSA/TIMP-2.

\section{Semi-quantitative analysis of Cy5.5-HSA/TIMP-2 accumulated in tumors}

The use of tumor biophotonic imaging with fluorescence-labeled therapeutics suffers from the fact that the quantitative data from whole organs are dramatically affected by organ size. After demonstrating that the Cy5.5-HSA/TIMP-2 fluorescent signal was inversely proportional to the tumor volume $\left(R^{2}=0.83\right.$; Fig. 3$)$, we generated a standard curve with various amounts of Cy5.5HSA/TIMP-2 that were intratumorally injected into different tumor volumes. The standard curve for the known amounts of Cy5.5-HSA/TIMP-2 in tumors versus the value of the fluorescence intensity multiplied by the tumor volume showed a strong correlation $\left(\mathrm{R}^{2}=0.92\right.$; Fig. $\left.4 \mathrm{~A}\right)$. To calculate the quantity of Cy5.5-HSA/TIMP-2 accumulated in a tumor, the fluorescence intensity from Figure 2B was multiplied by its tumor volume based on the standard curve. The amount of accumulated Cy5.5HSA/TIMP-2 was calculated to be $4.5 \pm 0.5 \mathrm{ng} / \mathrm{g}$ body weight in the tumor, representing $2.25 \pm$ $0.25 \%$ of the initial ip injection. The fluorescent signal at day 4 decreased significantly to $33.9 \%$ compared with that of day 2 (Fig. 4C, left panel), but the calculated amount of Cy5.5-HSA/TIMP-2 at day 4 was comparable to that of day 2 (Fig. 4C, right panel). These data suggested that the actual amount of accumulated Cy5.5-HSA/TIMP-2 showed retention in the tumor exceeding the fluorescent signal from the whole-body biophotonic imaging assay over time. 
A
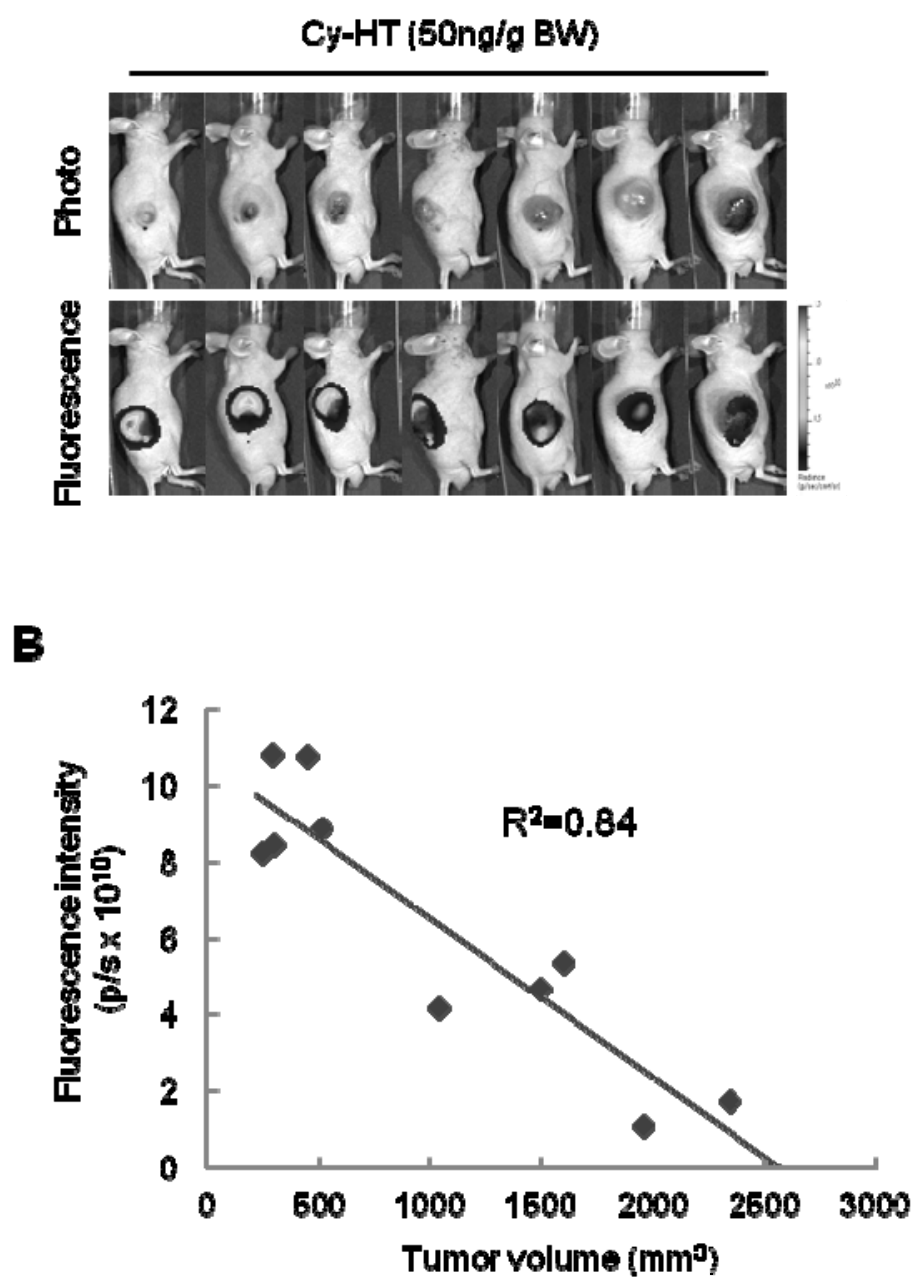

Figure 3. Correlation between Cy5.5 fluorescence intensity and tumor volume in MLL tumor xenografts. (A) Representative fluorescence image of MLL tumor xenografts. MLL cells ranging from $1 \times 10^{5}$ to $3 \times 10^{6}$ were implanted into the right dorsal flank of mice by subcutaneous (sc) injection to produce various tumor sizes. After 7 days, fluorescence images were acquired immediately following intratumoral injection of $50 \mathrm{ng} / \mathrm{g}$ body weight of Cy5.5-HSA/TIMP-2 in tumor xenografts $(n=10)$. At the same time, the tumor volume was measured using a caliper $\left(\mathrm{L} \times \mathrm{W}^{2} / 2\right)$. (B) Correlation between fluorescence intensity ( $\mathrm{p} / \mathrm{s}, y$-axis) and tumor volume $\left(\mathrm{mm}^{3}, x\right.$-axis; $\left.R^{2}=0.84\right)$. Cy-HT, Cy5.5-HSA/TIMP-2; BW, body weight.

The retained accumulation of Cy5.5-HSA/TIMP-2 at day 4 was confirmed by a comparable fluorescence signal of the Cy5.5-HSA/TIMP-2 observed in slices from the tumor by laser confocal microscopy (Fig. 5).

\section{DISCUSSION}

Our aim was to assess the PK and biodistribution of HSA/TIMP-2 using an NIR fluorescence imaging system in real time with intact living animals. To achieve this, we conjugated HSA/TIMP-2 with
Cy5.5 and tested Cy5.5-HSA/TIMP-2 in vitro and in vivo to verify that it was suitable for the present study. Cy5.5-HSA/TIMP-2 exhibited a relatively long blood half-life and was distributed preferentially in tumor masses in MLL xenografts, supporting previous reports that HSA fusion increases blood half-life and distribution in tumors $(17,18)$. Additionally, we could semi-quantify the actual amount of Cy5.5-HSA/TIMP-2 accumulated in tumors based on our standard curve, which provided a rapid and convenient method for examining the targeted drug in live tumors. 
A

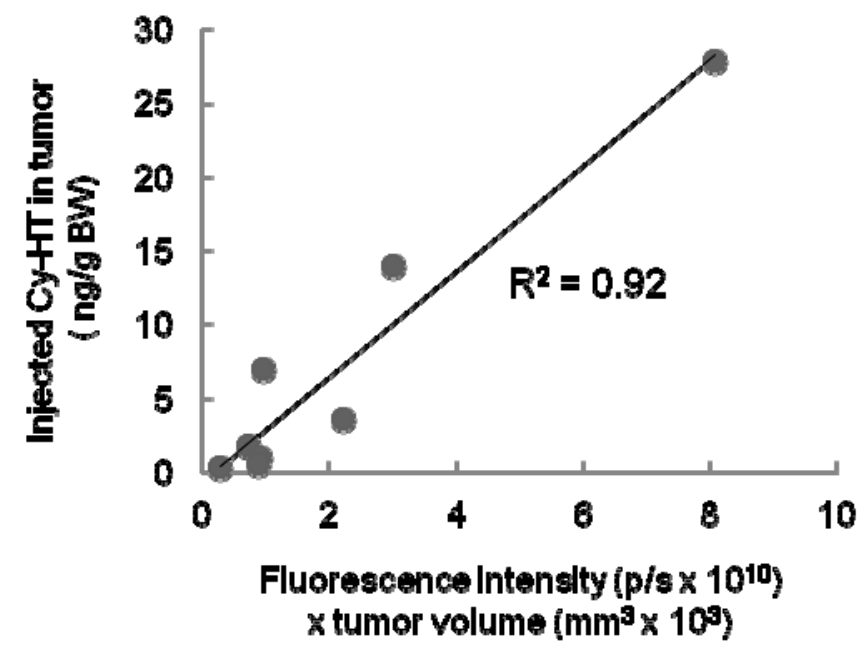

B
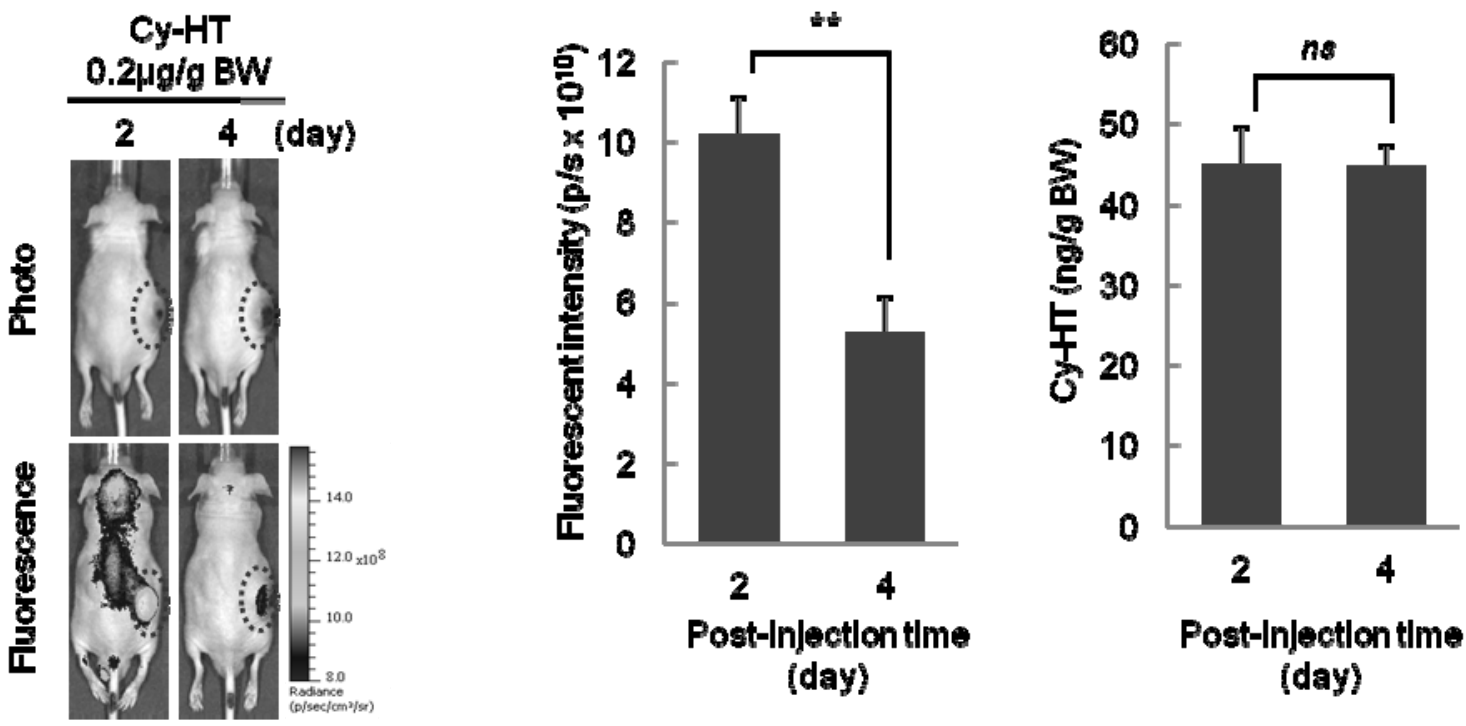

Figure 4. Semi-quantification of accumulated Cy5.5-HSA/TIMP-2 in the tumor. (A) Standard curve of fluorescence intensity multiplied by tumor volume versus known amounts of Cy5.5-HSA/TIMP-2 in the tumor. (B) Whole body images of MLL tumor xenografts at days 2 and 4 from Fig. 2A. (C) Quantitative comparison of fluorescence intensity (left panel) used to calculate the amount of Cy5.5-HSA/TIMP-2 (right panel). Based on the standard curve, the amount of accumulated Cy5.5-HSA/TIMP-2 was calculated from the fluorescent signal in the region of the tumor. Each data point represents the mean $\pm \operatorname{SEM}(n=4) .{ }^{*} P<0.05$ and ${ }^{* *} P<0.01$ by Student's $t$-test; $n s$, non-significant. Cy-HT, Cy5.5-HSA/TIMP-2; BW, body weight.

The use of optical imaging methods with an NIR fluorescent probe possesses advantages over conventional radiolabel imaging such as convenient labeling, fast lesion detection, and low tissue background (11). However, one limitation is inaccurate quantification due to light diffusion by tissue depth (13). In the current study, we proposed that the fluorescent signal was inversely proportional to the tumor volume. As shown in Supplementary data 2 , the correlation between the reduction in the fluorescent signal and the increase in tumor volume was high $\left(R^{2}=0.83\right)$. 


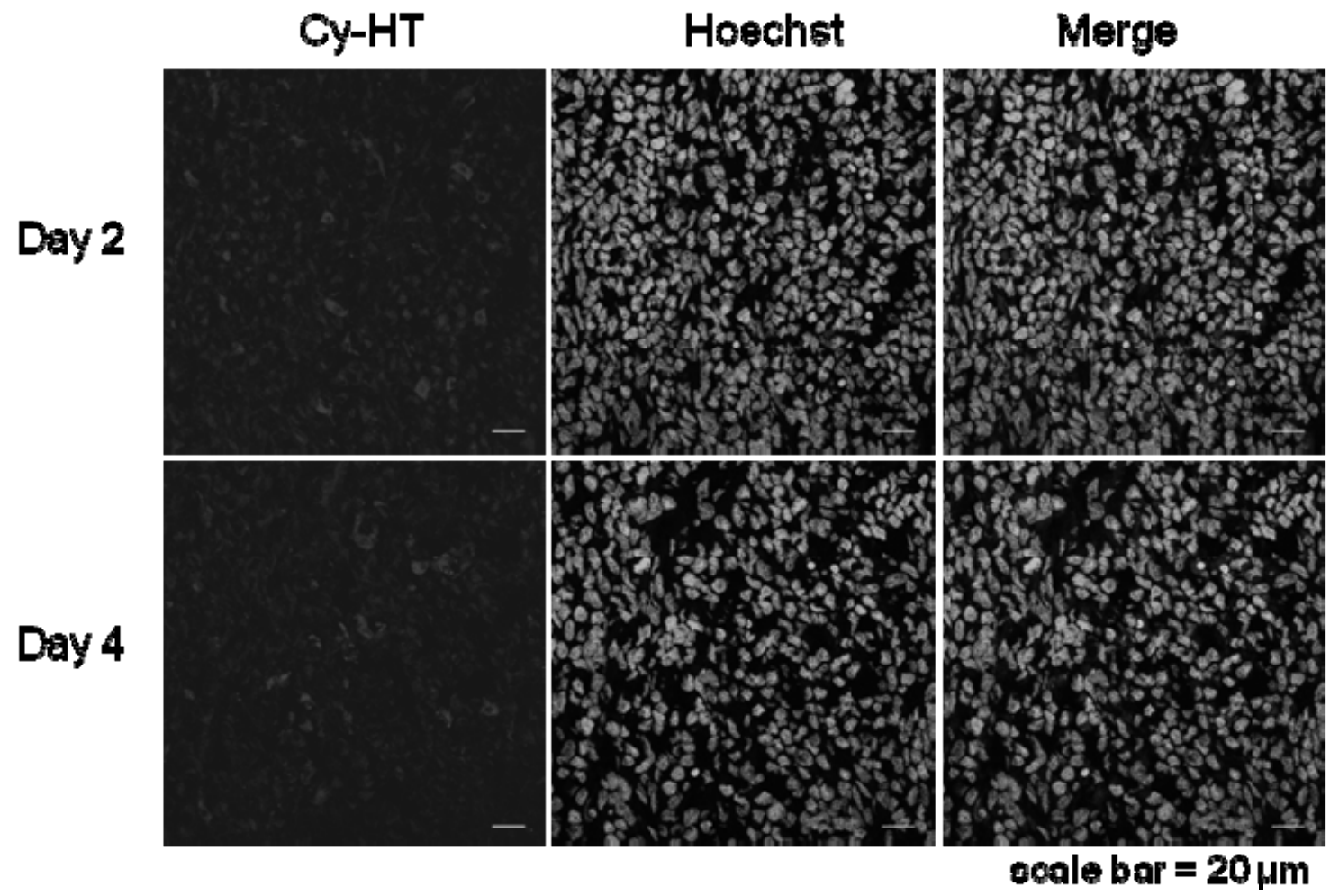

Figure 5. High-magnification image of tumor tissue from mouse administered Cy5.5-HSA/TIMP-2 at days 2 and 4. In tumor tissues, nuclei were labeled with Hoechst (blue). Note that the Cy5.5 signal (red) was generated within the cells (scale bar, $20 \mu \mathrm{m}$ ). Cy-HT, Cy5.5-HSA/TIMP-2.

Based on the results, we generated a standard curve, compensated by tumor volume, and calculated the accumulation of HSA/TIMP-2 in live tumors. Although several aspects, such as three-dimensional (3D) cancer geometry and the effects of nonuniform tumor tissue, should be carefully considered in developing a more accurate methodology, this new approach allows the semiquantification of Cy5.5-HSA/TIMP-2 targeted to the tumor without killing the animals.

HSA is a major component of protein in plasma and has been used as a therapeutic protein not only for prolonging the biological half-time of proteins in blood circulation (19) but also for increasing the yield of reluctant recombinant proteins in largescale production (20). Taking advantage of HSA fusion technology with the signal sequence of a mating factor secreted from $S$. cerevisiae, we could produce a high yield of the active HSA/TIMP-2 fusion protein in the culture broth.

The favorable PK and biodistribution properties of TIMP-2 due to HSA fusion in the current study have several possible mechanistic explanations. Initially, the increase in the molecular weight of
HSA/TIMP-2 (88 kDa) by the 67-kDa HSA fusion was thought to increase the circulatory half-life in blood circulation, where the glomerular filtration threshold is $60 \mathrm{kDa}$. Consistent with our results, the absolute amounts of tumor uptake of soluble synthetic macromolecules derived from copolymers of N-2-(hydroxypropyl)methacrylamide showed 0.5 $\pm 0.1 \%$ of injected dose/g tissue at $27.3 \mathrm{kD}$ and 1.2 $\pm 0.1 \%$ at $60.1 \mathrm{kDa}(21)$, suggesting size-dependent accumulation in tumors. High accumulation in tumors has also been explained by increased molecular weight through the effects of enhanced permeability and retention (22-24), resulting in much higher concentrations in tumors than in normal tissues and organs. In addition, our results agree with previous reports that albumin is preferentially taken up by tumor cells, which leads to high accumulation in tumor $(20,25,26)$. A related possibility is that the signal peptide of HSA (Fig. 1A) is also advantageous because it may enhance cytoplasmic delivery, leading to a higher cellular uptake of HSA/TIMP-2 (data not shown) and resulting in highly accumulated HSA/TIMP-2 in the tumor. 
Taken together, the PK and biodistribution data for HSA/TIMP-2 obtained using this quick and simple NIR optical imaging provided favorable results that complement studies of efficacy focused on tumor therapeutic applications of TIMP-2.

\section{ACKNOWLEDGMENTS}

This work was supported by a grant from the Korea Healthcare Technology R\&D Project, Ministry for Health, Welfare \& Family Affairs (grant no. A091037) and by a grant (kiom-2010-2) from the Inter-Institutional Collaboration Research Program under the Korea Research Council of Fundamental Science \& Technology (KRCF), Korea.

\section{REFERENCES}

1. Seo DW, Li H, Guedez L, Wingfield PT, Diaz T, Salloum R, Wei BY, Stetler-Stevenson WG. TIMP2 mediated inhibition of angiogenesis: an MMPindependent mechanism. Cell, 2003;114:171-180.

2. Ribatti D, Vacca A, Nico B, De Falco G, Giuseppe Montaldo P, Ponzoni M. Angiogenesis and antiangiogenesis in neuroblastoma. Eur $\mathrm{J}$ Cancer, 2002;38:750-757.

3. Fernandez CA, Butterfield C, Jackson G, Moses MA. Structural and functional uncoupling of the enzymatic and angiogenic inhibitory activities of tissue inhibitor of metalloproteinase-2 (TIMP-2): loop 6 is a novel angiogenesis inhibitor. J Biol Chem, 2003;278:40989-40995.

4. Baker AH, Wilkinson GW, Hembry RM, Murphy G, Newby AC. Development of recombinant adenoviruses that drive high level expression of the human metalloproteinase- 9 and tissue inhibitor of metalloproteinase-1 and -2 genes: characterization of their infection into rabbit smooth muscle cells and human MCF-7 adenocarcinoma cells. Matrix Biol, 1996;15:383-395.

5. Imren S, Kohn DB, Shimada H, Blavier L, DeClerck YA. Overexpression of tissue inhibitor of metalloproteinases-2 retroviral-mediated gene transfer in vivo inhibits tumor growth and invasion. Cancer Res, 1996;56:2891-2895.

6. Negro A, Onisto M, Masiero L, Garbisa S. Synthesis and refolding of human TIMP-2 from E. coli, with specific activity for MMP-2. FEBS Lett, 1995;360:52-56.

7. Kang WK, Park EK, Lee HS, Park BY, Chang JY, Kim MY, Kang HA, Kim JY. A biologically active angiogenesis inhibitor, human serum albuminTIMP-2 fusion protein, secreted from Saccharomyces cerevisiae. Protein Expression \& Purification, 2007;53:331-338.
8. Rossin R, Pan D, Qi K, Turner JL, Sun X, Wooley $\mathrm{KL}$, Welch MJ. 64Cu-labeled folate-conjugated shell cross-linked nanoparticles for tumor imaging and radiotherapy: synthesis, radiolabeling, and biologic evaluation. J Nucl Med, 2005;46:12101218.

9. Kaul G, Amiji M. Biodistribution and targeting potential of poly(ethylene glycol)-modified gelatin nanoparticles in subcutaneous murine tumor model. J Drug Target, 2004;12:585-591.

10. Lee MJ, Veiseh O, Bhattarai N, Sun C, Hansen SJ, Ditzler S, Knoblaugh S, Lee D, Ellenbogen R, Zhang M, Olson JM. Rapid pharmacokinetic and biodistribution studies using cholorotoxinconjugated iron oxide nanoparticles: a novel nonradioactive method. PLoS One, 2010;5:e9536.

11. Ntziachristos V. Fluorescence molecular imaging. Annu Rev Biomed Eng, 2006;8:1-33.

12. Li XD, O'Leary MA, Boas DA, Chance B, Yodh AG. Fluorescent diffuse photon density waves in homogeneous and heterogeneous turbid media: analytic solutions and applications. Appl Opt, 1996;35:3746-3758.

13. Dacosta RS, Wilson BC, Marcon NE. New optical technologies for earlier endoscopic diagnosis of premalignant gastrointestinal lesions. J Gastroenterol Hepatol, 2002;17 Suppl:S85-104.

14. Lee MS, Jung JI, Kwon SH, Shim I, Hahm DH, Han JJ, Han D, YoonPark JH, Her S. A New Bioluminescent Rat Prostate Cancer Cell Line: Rapid and Accurate Monitoring of Tumor Growth. J Life Sci, 2010;20:1738-1741.

15. Lee SJ, Park K, Oh YK, Kwon SH, Her S, Kim IS, Choi K, Lee SJ, Kim H, Lee SG, Kim K, Kwon IC. Tumor specificity and therapeutic efficacy of photosensitizer-encapsulated glycol chitosan-based nanoparticles in tumor-bearing mice. Biomaterials, 2009;30:2929-2939.

16. Lee MS, Kwon EH, Choi HS, Kwon SH, Lee CH, Shim IS, Lee SK, Her S. Quantification of cellular uptake and in vivo tracking of transduction using real-time monitoring. Biochem Biophys Res Commun, 2010;394:348-353.

17. Osborn BL, Olsen HS, Nardelli B, Murray JH, Zhou JX, Garcia A, Moody G, Zaritskaya LS, Sung C. Pharmacokinetic and pharmacodynamic studies of a human serum albumin-interferon-alpha fusion protein in cynomolgus monkeys. J Pharmacol Exp Ther, 2002;303:540-548.

18. Yeh P, Landais D, Lemaitre M, Maury I, Crenne JY, Becquart J, Murry-Brelier A, Boucher F, Montay G, Fleer R, et al. Design of yeast-secreted albumin derivatives for human therapy: biological and antiviral properties of a serum albumin-CD4 genetic conjugate. Proc Natl Acad Sci U S A, 1992;89:1904-1908. 
19. Zolle I, Rhodes BA, Wagner HN, Jr. Preparation of metabolizable radioactive human serum albumin microspheres for studies of the circulation. Int $\mathrm{J}$ Appl Radiat Isot, 1970;21:155-167.

20. Chuang VT, Kragh-Hansen U, Otagiri M. Pharmaceutical strategies utilizing recombinant human serum albumin. Pharm Res, 2002;19:569577.

21. Kissel M, Peschke P, Subr V, Ulbrich K, Schuhmacher J, Debus J, Friedrich E. Synthetic macromolecular drug carriers: biodistribution of poly[(N-2-hydroxypropyl)methacrylamide] copolymers and their accumulation in solid rat tumors. PDA J Pharm Sci Technol, 2001;55:191201.

22. Hamidi M, Azimi K, Mohammadi-Samani S. Coencapsulation of a Drug with a Protein in Erythrocytes for Improved Drug Loading and Release: Phenytoin and Bovine Serum Albumin (BSA). J Pharm Pharm Sci, 2011;14:46-59.
23. Matsumura Y, Maeda H. A new concept for macromolecular therapeutics in cancer chemotherapy: mechanism of tumoritropic accumulation of proteins and the antitumor agent smancs. Cancer Res, 1986;46:6387-6392.

24. Burger AM, Hartung G, Stehle G, Sinn H, Fiebig HH. Pre-clinical evaluation of a methotrexatealbumin conjugate (MTX-HSA) in human tumor xenografts in vivo. Int J Cancer, 2001;92:718-724.

25. Stehle G, Sinn H, Wunder A, Schrenk HH, Schutt S, Maier-Borst W, Heene DL. The loading rate determines tumor targeting properties of methotrexate-albumin conjugates in rats. Anticancer Drugs, 1997;8:677-685.

26. Kratz F, Roth T, Fichiner I, Schumacher P, Fiebig $\mathrm{HH}$, Unger C. In vitro and in vivo efficacy of acidsensitive transferrin and albumin doxorubicin conjugates in a human xenograft panel and in the MDA-MB-435 mamma carcinoma model. J Drug Target, 2000;8:305-318. 